\title{
El control interno en el proceso de contrataciones en las instituciones públicas
}

\author{
Sandra Ruíz Correa \\ sruiz_correa@hotmail.com
}

Dr. José Manuel Delgado Bardales

manueldelgado1506@hotmail.com

\section{RESUMEN}

El trabajo, tuvo como fin, conocer el control interno en el proceso de contrataciones en las instituciones del estado. Las variables principales son las contrataciones en el marco de un buen control interno, que tiene que ver con la protección de recursos públicos en el proceso de compras y licitaciones. La metodología de la investigación es de tipo aplicada, con diseño no experimental y de manera descriptiva, usando la revisión de artículos científicos y un método deductivo en el análisis respectivo, llegando a los siguientes resultados: El $30 \%$ de investigaciones mencionan que el control interno es aplicado por la autoridad competente mediante procedimientos desde el ambiente, riego, sistemas de información, actividades de control y seguimiento, con el objetivo de manejar eficientemente los recursos públicos y ayudar a cumplir con los objetivos de las instituciones públicas. Referente a las contrataciones con el estado, el $70 \%$ manifiesta que la normativa es confusa, muchos procedimientos que entrampan las contrataciones públicas aplicable y sencilla, liderada por el órgano de control interno, evitando infracciones y actos de corrupción en las licitaciones y compras. Concluyendo que el control interno es débil en las instituciones públicas, los procedimientos en cuanto a las contrataciones en compras y licitaciones no se desarrollan a cabalidad, a pesar de tener un aspecto vinculante, debido a que lo procedimientos son poco claros y confusos, llevando a que el órgano de control tenga que llegar a sancionar a las instituciones. Es decir, falta estandarizar los procesos y hacerlos más sencillos, con el objetivo de que las instituciones cumplan los objetivos usando los recursos de manera eficiente.

Palabras clave: Control interno, Contracciones con el estado, contratos, licitaciones, compras, eficiencia. 


\title{
Internal control in the process of hiring in public institutions
}

\begin{abstract}
The purpose of the work was to learn about internal control in the hiring process in state institutions. The main variables are contracting within the framework of good internal control, which has to do with the protection of public resources in the procurement and bidding process. The research methodology is of an applied type, with a non-experimental design and in a descriptive way, using the review of scientific articles and a deductive method in the respective analysis, reaching the following results: $30 \%$ of investigations mention that internal control It is applied by the competent authority through procedures from the environment, irrigation, information systems, control and monitoring activities, with the aim of efficiently managing public resources and helping to meet the objectives of public institutions. Regarding contracting with the state, $70 \%$ state that the regulations are confusing, many procedures that trap public contracting applicable and simple, led by the internal control body, avoiding infractions and acts of corruption in bids and purchases. Concluding that internal control is weak in public institutions, the procedures regarding procurement in purchases and tenders are not fully developed, despite having a binding aspect, because the procedures are unclear and confusing, leading to that the control body has to get to sanction the institutions. In other words, it is necessary to standardize the processes and make them simpler, in order for the institutions to meet the objectives by using resources efficiently.
\end{abstract}

Keywords: Internal control, Contractions with the state, contracts, tenders, purchases, efficiency.

Artículo recibido: 03 nov. 2020 Aceptado para publicación: 07 dic. 2020 Correspondencia: sruiz_correa@hotmail.com. Conflictos de Interés: Ninguna que declarar 


\section{INTRODUCCIÓN}

En la mayoría de estados del mundo, el $70 \%$ de su presupuesto es gastado en contrataciones o adquisiciones de bienes o servicios; sin embargo, en estos procesos se presentan indicios de corrupción, perjudicando seriamente a los ciudadanos, debido a que los fondos destinados para el desarrollo del país pasan a bolsillos de algunos funcionarios. Estos problemas se han presentado con mayor frecuencia en países de América Latina, con grandes escándalos de corrupción que desacredita la imagen de las instituciones públicas (Transparency internacional, 2018, p. 3). Así mismo, la mayoría de países tienen problemas en el control interno de las instituciones públicas; la misma que estudia la información financiera fraudulenta en las mismas y que no se aplican a tiempo para prevenir problemas de corrupción a grandes escalas (Cuevas, 2018, p.2).

El Perú, cuenta con un Organismo Supervisor de las Contrataciones del Estado (OSCE), adscrito al Ministerio de Economía y Finanzas, tiene por objetivo con un plan respectivo para fiscalizar todos los procesos de contrataciones sean transparentes y evitar asuntos de corrupción (OSCE, 2019). A su vez, también cuenta con la contraloría general de la república, organismo de brindar soporte y asesoramiento respecto al uso de recursos públicos (Contraloría general de la república, 2014, p.6).

Sin embargo, faltan potenciar los procesos de control desde las contrataciones ya que existen casos de corrupción en los diferentes niveles de gobierno, por la que se necesaria implementar herramientas de control para salvaguardar los recursos públicos.

En San Martín, el gobierno regional debido a algunos hechos de corrupción, se ha incluido dentro del seguimiento de investigación administrativa, fiscal y cumplimiento de obligaciones del gobierno por el deficiente control interno y la escasa administración de los recursos financieros. A esto se suma la corrupción en las contrataciones con el estado, donde al final las obras públicas terminan sobrevaloradas, con adicionales y mal hechas, retrasando el desarrollo social y económico.

\section{Teorías de las variables}

Control interno

El control interno, está referido a un proceso de actividades que se desarrollan con eficiencia, con el fin de cumplir con los objetivos de las instituciones. A su vez, es considerado como una herramienta de apoyo administrativo para evaluar periódicamente el desempeño organizacional, tomando acciones oportunas y reducir cuellos de botellas y 
posibles problemas posteriores (Romero, 2012, p. 35). A su vez, Chacón (2018), indica que el control interno, tiene políticas, normas y procedimientos, con el fin de lograr las metas organizacionales; así mismo, funciona como una herramienta importante para direccionar, asegurar y garantizar el cumplimiento de objetivos; así mismo, previene fraudes, desviaciones y salvaguarda los recursos (p. 55).

Así mismo el control interno es importante porque permite supervisar y fiscalizar las actividades de manera periódica de las instituciones, detectando errores a tiempo y corregirlos, con ello se evita el uso irracional y desperdicio de recursos (Perdomo, 2004, p.34). Quiere decir, que el control interno, da facilidades a las instituciones para cumplir con sus obligaciones, salvaguardando los recursos públicos de fraudes o actos de corrupción; aparte que les brinda herramientas de planificación, ejecución y seguimiento para el buen control de recursos.

Proceso de contrataciones con el estado.

Respecto al proceso de contrataciones con el estado, se refiere a la adquisición de bienes y servicios mediante licitaciones, reconocido como un acto dentro de la economía de mercado; la misma, está sujeta a un proceso de normas, donde existe un pago de por medio (Huamán y Jiménez, 2014, p. 25). La ley de contrataciones es una norma en la que determina los lineamientos de contrataciones y adquisiciones, tanto en compras o ejecución de obras en los diferentes niveles de gobierno (Campos, 2014, p. 14).

También es importante tener en cuenta los términos de referencia en las licitaciones, ya que son indispensables para realizar una correcta contratación con el estado. Estos deben estar definidos de forma clara y precisa respecto a los servicios que se necesitan contratar, con los plazos y requisitos mínimos del proveedor con el personal, entre otros aspectos a considerar.

La descripción del problema a nivel internacional, nacional y local, nos vislumbra el planteamiento del problema, pasando a formular el siguiente problema de investigación: ¿Cómo es el control interno en las contrataciones en las instituciones públicas?

A su vez, se tiene el siguiente objetivo de investigación: Conocer y proponer mejoras en el control interno de las contrataciones en las instituciones públicas.

Dentro del propósito, la investigación busca conocer como es el control interno y su influencia en las contrataciones, sus características, elementos; con eso, proponer mejoras para el desarrollo de las instituciones públicas, mediante las conclusiones. 
Como justificaciones se encuentran las siguientes:

Por conveniencia, las conclusiones serán de gran utilidad como insumo para que las autoridades o tomadores de decisiones, implementen políticas, con herramientas eficientes para mejorar el control interno en las contrataciones con el estado de las instituciones públicas.

La justificación social, se basa en que beneficiará a los trabajadores de las instituciones públicas a tener implicancias por malos manejos en las contrataciones. Así mismo, ayudará a salvaguardar los recursos públicos y ello conllevará al desarrollo social y económico; así mismo, ayudará a tener instituciones transparentes, fortaleciendo la imagen que tiene los ciudadanos respecto al manejo de los recursos públicos.

La justificación teórica, se basa en la utilización de teorías mencionadas por los autores de las diferentes investigaciones utilizadas, respecto al control interno y contrataciones con el estado, que servirán de referencia para otras investigaciones con el mismo contenido.

La justificación práctica, está enfocada en solucionar las contrataciones con el estado mediante un control interno eficiente, que ayude a salvaguardar los recursos públicos; todo esto a través de la implementación de políticas, con herramientas necesarias para lograr mejorar al respecto.

Metodológicamente, la investigación mediante la metodología descriptiva, cualitativa y la forma de recolección de datos mediante la revisión sistemática, ayudará a otras investigaciones similares dentro de la comunidad científica.

\section{Material y métodos}

El tipo de investigación es cualitativa, con diseño no experimental y descriptivo, con revisiones sistemáticas de estudios primarias de artículos científicos de revistas nacionales e internacionales. Como técnica se utilizó el análisis bibliográfico, y como instrumento la guía de análisis sistemática.

En los materiales, se usó laptop, cuadros de análisis y bibliografía especializada de investigaciones para la realización de la investigación.

El método utilizado se partió de hechos particulares que corresponde al método analítico inductivo para llegar a hechos generales mediante las conclusiones finales.

La recolección de datos se realizó a través de las diferentes investigaciones utilizadas, con autores nacionales e internacionales de revistas indexadas de los últimos 5 años, donde se tuvo como principal tema, el control interno y las contrataciones con el estado de las 
instituciones públicas, teniendo en cuenta el grado de corrupción dentro de la pequeña, mediana y gran escala de nuestras entidades del estado, realizando el análisis siempre y cuando se tuvo acceso al tex to completo de las investigaciones.

Después de la recolección de datos, se realizó el análisis de resultados correspondiente a cada investigación, mostrada en las tablas de análisis, teniendo en cuenta las variables de estudio, donde se mostró a los autores, título, Link de la revista y el nombre de la misma; a su vez, se mostró el desarrollo donde se colocó la metodología, resultados y conclusiones principales para ser analizadas.

Luego se procedió al análisis de la información presentada, con las principales conclusiones de los diferentes autores presentados; se procedió a discutir los resultados, realizando discrepancias similitudes entre la información encontrada en cada investigación; siendo un elemento principal para realizar las conclusiones generales del trabajo y comparar el objetivo presentado.

La ética de investigación tuvo en cuento algunos aspectos considerados por el investigador y la revista. A su vez, se tuvo en cuenta las citas correspondientes de los autores respectivos.

\section{Resultados.}

Los resultados se presentan en las tablas respectivas con el contenido necesario como autor/es, título, ubicación de la revista, metodología, conclusiones y principales recomendaciones, descritas a continuación. 
Tabla 1

Datos de investigación 1

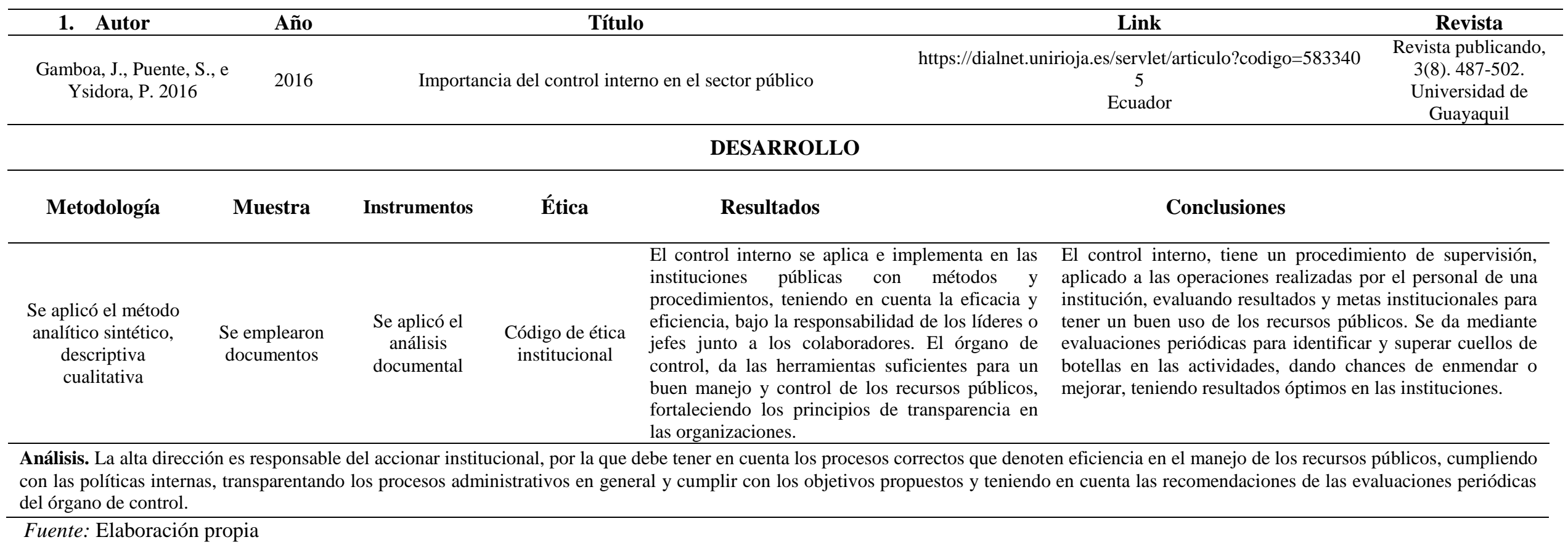

Fuente: Elaboración propia

Tabla 2

Datos de autor 2

\begin{tabular}{|c|c|c|c|c|}
\hline 2. $\quad$ Autor & Año & Título & Link & Revista \\
\hline $\begin{array}{l}\text { Mendoza, W., García, T., } \\
\text { Delgado, M., y Barreiro, I. }\end{array}$ & 2018 & $\begin{array}{l}\text { El control interno y su influencia } \\
\text { en la gestión administrativa del } \\
\text { sector público }\end{array}$ & https://dialnet.unirioja.es/servlet/articulo?codigo $=6656251$ & $\begin{array}{l}\text { Revista Dom. Cien, Vol. 4, } \\
\text { núm.4., oct, pp. 206-240 }\end{array}$ \\
\hline \multicolumn{5}{|c|}{ DESARROLLO } \\
\hline Metodología & Muestra & Instrumentos & Resultados & Isiones \\
\hline
\end{tabular}

1339Ciencia Latina Revista Científica Multidisciplinar, Ciudad de México, México.

ISSN 2707-2207 / ISSN 2707-2215 (en línea), julio-diciembre, 2020, Volumen 4, Número 2.

https://doi.org/10.37811/cl rcm.v4i2.159 p. 1339 
analítico en materia de

análisis

actividades internas para el logro de recursos públicos, refuerza los sistemas objetivos. Está integrada por, lo integran administrativos que tiene que ver con la todos los organismos del poder público. eficiencia del gasto desde la formulación

Tiene como ponentes del entorno ambiente, y ejecución de compras, logística y de riesgo, de sistemas de información, almacén.

actividades de control y seguimiento en las instituciones

Análisis: El control interno ayuda alas instituciones a utilizar correctamente los recursos, de manera transparente y eficaz; brinda las herramientas para que los trabajadores refuercen sus labores con mejores sistemas administrativos que intervienen en los procesos de compras y logística, evitando que los recursos sean desviados a objetivos que no tienen que ver con la institución.

Fuente: Elaboración propia

Tabla 3

Datos de autor 3

\begin{tabular}{|c|c|c|c|c|c|}
\hline 3. Autor. & Año & \multicolumn{2}{|r|}{ Título } & \multirow{2}{*}{\multicolumn{2}{|c|}{$\begin{array}{l}\text { Volumen y número. } \\
\text { evista Cofín Habana. } \\
\text { 2018. 12. (Número 1). } \\
\text { 268-283 }\end{array}$}} \\
\hline $\begin{array}{l}\text { Quinaluisa, N., Ponce, V. } \\
\text { Muñóz, C., y otros }\end{array}$ & 2018 & $\begin{array}{l}\text { El control interno } \\
\text { COSO y COCO }\end{array}$ & sus herramientas de aplice & & \\
\hline \multicolumn{6}{|c|}{ CUERPO } \\
\hline Metodología & Muestra & Instrumentos & Ética & Resultados & Conclusiones \\
\hline $\begin{array}{l}\text { No experimental } \\
\text { y descriptivo }\end{array}$ & $\begin{array}{l}\text { Documentaos } \\
\text { bibliográficos }\end{array}$ & Análisis bibliográfico & 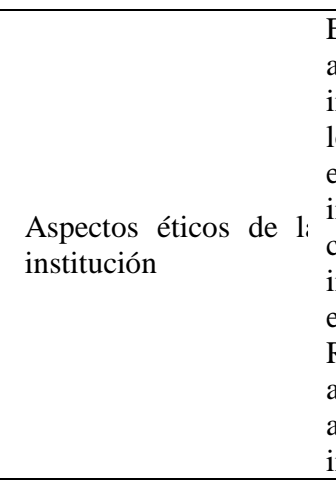 & $\begin{array}{l}\text { El control interno en los países, ha ayudado } \\
\text { a mejorar algunos problemas e } \\
\text { inconformidades, mejorando la confianza de } \\
\text { los ciudadanos respecto al control y } \\
\text { ejecución de recursos. Mas del } 80 \% \text { de } \\
\text { instituciones cuentan con sistemas de } \\
\text { control eficaces que minimizan costos e } \\
\text { incrementan logros de objetivos; sin } \\
\text { embargo, no se han aplicado correctamente. } \\
\text { Respecto a los informes COSO y COCO, } \\
\text { ambos tienen diferentes enfoques, pero } \\
\text { ayudan al control interno de las } \\
\text { instituciones. }\end{array}$ & $\begin{array}{l}\text { El control interno, ayuda a controlar el uso de recursos } \\
\text { salvaguardándolos. Permite cumplir con las metas y } \\
\text { objetivos de manera eficaz y eficiente. El COSO y COCO } \\
\text { ayuda a mejorar la rendición de cuentas y arreglar } \\
\text { problemas y aprovechar los recursos al máximo, evitando } \\
\text { desperdicios o tentativas ilícitas. }\end{array}$ \\
\hline
\end{tabular}

Ciencia Latina Revista Científica Multidisciplinar, Ciudad de México, México. ISSN 2707-2207 / ISSN 2707-2215 (en línea), julio-diciembre, 2020, Volumen 4, Número 2. https://doi.org/10.37811/cl_rcm.v4i2.159 p. 1340 


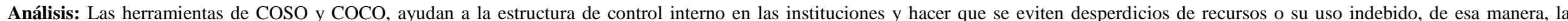
institución cumple con los objetivos y metas propuestas. Ambas herramientas son eficientes y similares.

Fuente: Elaboración propia

Tabla 04

Datos de publicación de autor 04.

4. Autor.

Año

Título

Ubicación de la revista

Volumen y número.

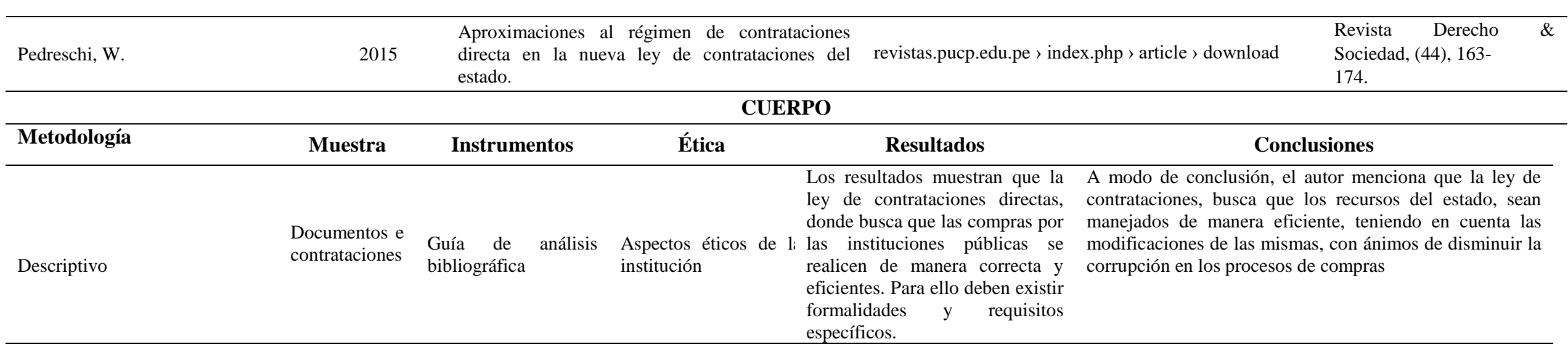

Análisis: La ley de contrataciones directas, están relacionadas con las compras que realizan las instituciones de manera eficiente, con un buen control interno, incluyendo formalidades y procedimientos correctos en las compras.

Fuente: Elaboración propia

Ciencia Latina Revista Ciencia Latina Revista Científica Multidisciplinar, Ciudad de México, México. ISSN 2707-2207 / ISSN 2707-2215 (en línea), julio-diciembre, 2020, Volumen 4, Número 2. 
Tabla 05:

Datos de investigación 05

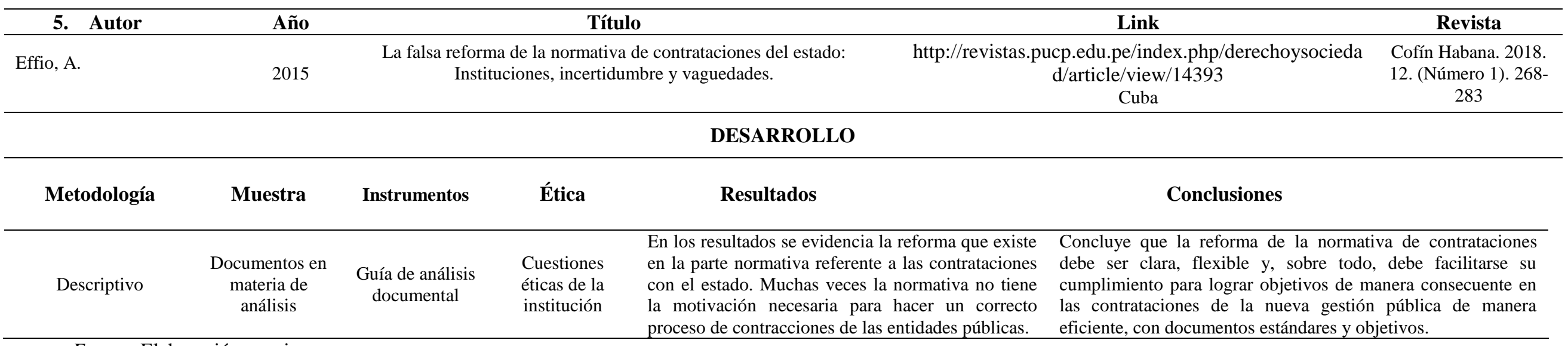

Fuente: Elaboración propia.

Tabla 06

Dataos de publicación de autor 06

\begin{tabular}{|c|c|c|c|c|}
\hline 6. $\quad$ Autor & Año & Título & Ubicación de la revista & Volumen y número \\
\hline Franco, B. & 2015 & $\begin{array}{l}\text { El carácter vinculante de las opiniones del } \\
\text { organismo supervisor de las contrataciones del } \\
\text { estado }\end{array}$ & $\begin{array}{l}\text { http://revistas.pucp.edu.pe/index.php/derecho\%20ysociedad } \\
\text { /article/view/14420 } \\
\text { Perú }\end{array}$ & $\begin{array}{l}\text { Revista Derecho \& } \\
\text { Sociedad, (44), 391-402. }\end{array}$ \\
\hline
\end{tabular}

\section{DESARROLLO}

\begin{tabular}{|c|c|c|c|c|c|}
\hline Metodología & Muestra & Instrumentos & $\begin{array}{l}\text { Ética de la } \\
\text { institución }\end{array}$ & Resultados & Conclusiones \\
\hline Descriptivo & $\begin{array}{l}\text { Documentos } \\
\text { para } \\
\text { normativos de } \\
\text { contrataciones }\end{array}$ & $\begin{array}{l}\text { Guía de análisis } \\
\text { documental }\end{array}$ & $\begin{array}{l}\text { Ética } \\
\text { investigador }\end{array}$ & $\begin{array}{l}\text { La investigación mostró la descripción } \\
\text { normativa y las opiniones que emiten el } \\
\text { órgano que supervisa las contrataciones con } \\
\text { el estado. A su vez, menciona los elementos }\end{array}$ & $\begin{array}{l}\text { Concluye que las entidades carecen de } \\
\text { credibilidad al pedir una opinión al OSCE, } \\
\text { teniendo en cuenta que la opinión del mismo es la } \\
\text { última palabra y es irrevocable cuando quieren }\end{array}$ \\
\hline
\end{tabular}

Ciencia Latina Revista Científica Multidisciplinar, Ciudad de México, México.

ISSN 2707-2207 / ISSN 2707-2215 (en línea), julio-diciembre, 2020, Volumen 4, Número 2. https://doi.org/10.37811/cl rcm.v4i2.159 p. 1342 
vinculantes en materia de interpretación de la las entidades volver o ignorar lo dicho por este norma. Incluso, muestra las características de ente.

la función administrativa en diferentes etapas

del proceso de contrataciones públicas.

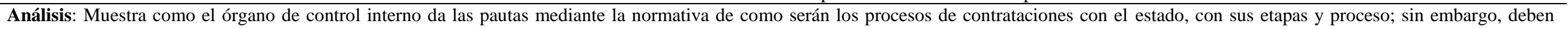
resolver las dudas consultadas por las entidades, evitando así cometer errores en los procesos de compras.

Fuente: Elaboración propia.

Tabla 07

Datos de investigación 07

\begin{tabular}{|c|c|c|c|c|}
\hline 7. Autor & Año & Título & Revista donde se ubica la Publicación & Volumen Y Numero \\
\hline Becerra, M. & 2016 & $\begin{array}{l}\text { El tribunal de contrataciones del estado en el } \\
\text { periodo 2012-2014. }\end{array}$ & $\begin{array}{l}\text { http://revistas.pucp.edu.pe/index.php/derechoysociedad/artic } \\
\text { le/view/14417/15031 } \\
\begin{array}{l}\text { Perú }\end{array}\end{array}$ & $\begin{array}{l}\text { Revista } \quad \text { Derecho } \\
\text { Sociedad, (44), 353-363 }\end{array}$ \\
\hline
\end{tabular}

\section{DESARROLLO}

\begin{tabular}{|c|c|c|c|c|c|}
\hline Tipo de investigación & Muestra & Instrumentos & Ética & Resultados & Conclusiones \\
\hline Descriptiva & $\begin{array}{c}\text { Documentos } \\
\text { de } \\
\text { contrataciones }\end{array}$ & $\begin{array}{l}\text { Guía de análisis } \\
\text { normativo }\end{array}$ & Ética de investigador & $\begin{array}{l}\text { Como resultados, se tuvo que con la } \\
\text { implementación de la ley de } \\
\text { contrataciones desde el } 2012 \text { al } \\
\text { 2014. Incrementándose la carga } \\
\text { procesal, incrementándose el tiempo } \\
\text { de atención que inciden } \\
\text { positivamente y de manera eficiente } \\
\text { en los procesos de compras de las } \\
\text { instituciones gubernamentales. Sin } \\
\text { embargo, es necesario que la entidad } \\
\text { competente institucionalice y } \\
\text { estandarice los procesos, que sean } \\
\text { igual para todos. }\end{array}$ & $\begin{array}{l}\text { Concluye que, la ley de contrataciones desde el } 2012 \text { al } \\
2014 \text {, se denota que creció la corrupción en un buen } \\
\text { porcentaje de acuerdo al cuadro estadístico y existiendo } \\
\text { poca capacidad operativa para dar respuesta a través de } \\
\text { las opciones del OSCE. }\end{array}$ \\
\hline
\end{tabular}

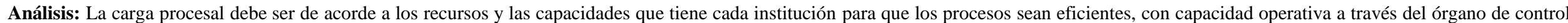
interno; también es necesario la estandarización de procesos que sean aplicados para todos de manera uniforme e igual.

Fuente: Elaboración propia

Ciencia Latina Revista Ciencia Latina Revista Científica Multidisciplinar, Ciudad de México, México. ISSN 2707-2207 / ISSN 2707-2215 (en línea), julio-diciembre, 2020, Volumen 4, Número 2.

https://doi.org/10.37811/cl rcm.v4i2.159 p. 1343 
Tabla 08

Datos de investigación 08

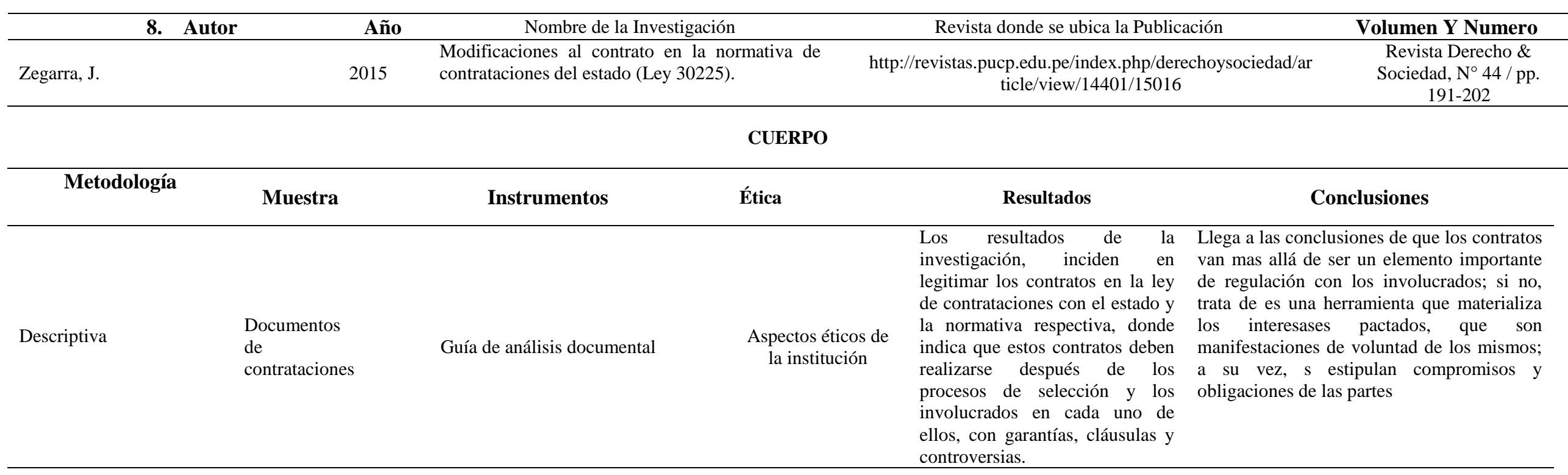

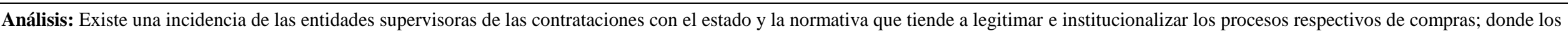
contratos deben ser considerados después de los procesos de selección, con garantías, cláusulas y condiciones entre las partes.

Fuente-. Elaboración propia

Tabla 09

Datos de investigación 09

\begin{tabular}{|c|c|c|c|c|}
\hline 9. $\quad$ Autor & Año & Nombre & Ubicación de revista & Volumen y número \\
\hline Castillo, M., y Sabroso, M. & 2019 & $\begin{array}{l}\text { Los árbitros y el deber de revelación en la ley } \\
\text { de contrataciones del estado y su reglamento }\end{array}$ & $\begin{array}{l}\text { http://revistas.pucp.edu.pe/index.php/derechoysociedad/ } \\
\text { article/view/14408/15022 } \\
\text { Perú }\end{array}$ & $\begin{array}{l}\text { Revista Derecho } \\
\text { Sociedad, (44), 245-255. }\end{array}$ \\
\hline
\end{tabular}

Ciencia Latina Revista Científica Multidisciplinar, Ciudad de México, México.

ISSN 2707-2207 / ISSN 2707-2215 (en línea), julio-diciembre, 2020, Volumen 4, Número 2. https://doi.org/10.37811/cl rcm.v4i2.159 p. 1344 


\section{DESARROLLO}

\begin{tabular}{|c|c|c|c|c|c|}
\hline Metodología & Muestra & Instrumentos & Ética & Resultados & Conclusiones \\
\hline Descriptivo & $\begin{array}{l}\text { Documentos } \\
\text { legales }\end{array}$ & $\begin{array}{l}\text { Guía de revisión } \\
\text { documentaria }\end{array}$ & $\begin{array}{l}\text { Ética de } \\
\text { investigac } \\
\text { ión de la } \\
\text { institución }\end{array}$ & $\begin{array}{l}\text { En los resultados muestra los requisitos que se } \\
\text { aplican en los procesos de contratación especiales, } \\
\text { donde debe existir un árbitro para mediar dicho } \\
\text { proceso. A su vez, tipifica las obligaciones que } \\
\text { tienen, sin mantener ninguna relación con las partes. } \\
\text { Todo esto lo realiza la OSCE, la parte ética que rige } \\
\text { para todas para partes en el proceso de } \\
\text { contrataciones. }\end{array}$ & $\begin{array}{l}\text { Concluye que, en los procesos de contrataciones especiales, } \\
\text { intervienen los árbitros, con obligaciones de acuerdo al reglamento } \\
\text { de la ley de contrataciones. Si embargo, casi siempre cuando llega } \\
\text { al tribunal a través de los árbitros existe mucha duda al momento } \\
\text { de dar un resultado final del arbitraje siendo casi siempre el } \\
\text { Contratista existiendo un problema mayor para la Entidad, porque } \\
\text { tiene que someterse a lo solicitado por Contratista y ganado a } \\
\text { través de los Árbitros. }\end{array}$ \\
\hline
\end{tabular}

Análisis: Se tuvo en cuenta en que las contrataciones especiales se realizan con la presencia de un árbitro en los procesos de compras; los mismo tienen obligaciones, sin relacionarse con los involucrados y a la ética que les corresponde a todos.

\section{Fuente: Elaboración propia}

Tabla 10

Datos de publicación de investigación 10

\section{Autor \\ Año}

Rivas, F., Asprino, M., Sarache, J., y

otros

2019

Sistema inteligente para la generación automática

contratos en el marco de la ley de contrataciones públicas.
Ubicación de revista

https://doi.org/10.33333/rp.vol44n1.05 Ecuador
Volumen y número

Revista Politécnica, Vol. 44, No. 1

DESARROLLO

\begin{tabular}{|c|c|c|c|c|c|}
\hline Metodología & Muestra & Instrumentos & Ética & Resultados & Conclusiones \\
\hline $\begin{array}{l}\text { Descriptivo } \\
\text { analítico }\end{array}$ & $\begin{array}{l}\text { Documentos } \\
\text { en materia } \\
\text { de análisis }\end{array}$ & Cuestionario & $\begin{array}{l}\text { Código de ética } \\
\text { de la entidad }\end{array}$ & $\begin{array}{l}\text { En los resultados se evidencia la normativa que } \\
\text { establece el correcto proceso de generación automática } \\
\text { de contratos en el marco de la ley de contrataciones } \\
\text { gubernamentales. Dentro de ello, podemos encontrar el } \\
\text { objeto del contrato, montos, aportes sociales y otras } \\
\text { especificaciones enmarcadas en la ley. }\end{array}$ & $\begin{array}{l}\text { Llega a la conclusión de que existen páginas web y } \\
\text { herramientas de creación de contratos de manera } \\
\text { automática, como técnica de inteligencia artificial, } \\
\text { adaptando requerimientos de manera sencilla y } \\
\text { haciendo mas eficiente el proceso de contrataciones } \\
\text { para la adquisición de bines y obras en las } \\
\text { instituciones. }\end{array}$ \\
\hline
\end{tabular}

Análisis: Las herramientas digitales deben ser un soporte para generar automáticamente los contratos de compras públicas, enmarcados en la ley de contrataciones con el estado; donde se estipulan las características de los mismo; con ello, se ayuda facilitar los procesos de contrataciones.

Fuente: Elaboración propia

Ciencia Latina Revista Ciencia Latina Revista Científica Multidisciplinar, Ciudad de México, México. ISSN 2707-2207 / ISSN 2707-2215 (en línea), julio-diciembre, 2020, Volumen 4, Número 2. https://doi.org/10.37811/cl rem.v4i2.159 p. 1345 


\section{DISCUSIÓN}

Después de haber analizado los resultados de cada autor, nos permite conocer el control interno de las contrataciones en las instituciones públicas. Lo cual resulta que el $30 \%$ de investigaciones mencionan que es aplicado por la autoridad competente mediante procedimientos desde el ambiente, riego, sistemas de información, actividades de control y seguimiento, con el objetivo de manejar eficientemente los recursos públicos y ayudar a cumplir con los objetivos de las instituciones. Mas del $80 \%$ de las instituciones cuentan con organismos de control eficaces; sin embargo, siempre buscan sacar la vuelta al estado en las contrataciones con el estado referente a compras y manejo logístico, muchas veces se da por las escasas respuestas que da OSCE y la poca claridad de la normativa.

Referente a las contrataciones con el estado, el $70 \%$ manifiesta que la normativa es confusa, muchos procedimientos que entrampan las contrataciones públicas aplicable y sencilla, liderada por el órgano de control interno, evitando infracciones y actos de corrupción en las licitaciones y compras. La ley de contrataciones, al tener un aspecto vinculante, ayuda a crear procesos eficientes, cuidando así los recursos del estado; sin embargo, estos deben ser estandarizados y aplicado de manera uniforme para todos y evitar sanciones por parte del órgano de control.

En ese marco Gamboa, Puente e Ysidora (2016), concuerda que el control interno es aplicado en todas las instituciones del estado, para ello se deben brindar todas las herramientas necesarias para un buen manejo de los recursos públicos. Por su parte, Mendoza, García y Barrieiro (2018), concuerda en que el control interno direcciona las actividades de las instituciones, ayudándoles a cumplir sus metas, siguiendo un conjunto de procedimientos de ambiente de control, riesgos, sistemas de información y seguimiento respectivo a la ejecución de los recursos públicos.

Sin embargo, Quinaluisa, Ponce, Muñoz y otros (2018), discute que no sólo se trata de seguir ciertos lineamientos tradicionales para cumplir con el control interno, si no también se puede aplicar herramientas electrónicas como COSO y COCO, que facilitan los procedimientos, haciéndolos menos costos y accesibles. También Effio (2015), concuerda con los resultados referentes a que la ley de contrataciones y su reforma, debe ser entendible y flexible, que facilite su aplicación y cumplimiento |de forma eficiente.

También concuerda, Pedrechi (2015), que la ley de contrataciones tiene el objetivo de evitar la corrupción en los procesos de compras; de esa forma, manejar correctamente los 
recursos del estado. Contrariamente, Franco (2015), indica que muchas entidades públicas, no tienen credibilidad al pedir opinión al órgano de control OSCE, ya que generalmente no aplican lo recomendado, o tienden a ignorar dichas opiniones. De la misma forma, Becerra (2016), concuerda que la ley de contrataciones durante el periodo 2012 al 2014, la norma tuvo escasa capacidad operativa para responder a través del órgano de control OSCE, haciendo que los casos de corrupción por compras y licitaciones vaya en aumento.

Por su parte, Zegarra (2015) concuerda con los resultados en que los contratos son importantes, que no solo contemplan la regulación de las partes, si no que, ayuda a materializar los intereses estipulados y pactados, dejando ver la voluntad de los involucrados, con los respectivos compromisos y obligaciones. A su vez, Castillo, y Sabroso (2019), concuerdan con el 70\% de autores, que el tipo de contrataciones especiales, se necesitan intermediarios como son los árbitros, que no tienen relaciones personales con ninguna de las partes. Los mismos se rigen en el reglamento de contrataciones, con obligaciones y respuestas finales contundentes, deslindando las dudas de las entidades y haciendo que el estado salga ganando.

Por último, Rivas, Aspirino, Sarache y otros (2019), concuerdan con el 70\% de autores en que existen herramientas digitales que agilizan y mejoraran la eficiencia en la ceración de contratos en línea de manera rápida y automática, facilitando el proceso de contrataciones con el estado y la adquisición de bienes y servicios por las entidades públicas.

\section{CONCLUSIONES}

Con las investigaciones presentadas, se buscó conocer las características del control interno en las contrataciones con el estado de las instituciones públicas. Las investigaciones fueron a través de artículos científicos de revistas a nivel internacional y nacional, con metodología descriptiva, lo que ayudó a llegar a las siguientes conclusiones.

El control interno es aplicado mediante un conjunto de procedimientos en todas las instituciones públicas, con el objetivo de salvaguardar los recursos públicos, a su vez, brinda las herramientas administrativas a todos los colaboradores para el control y seguimiento de actividades que permitan cumplir con los objetivos institucionales. En la mayoría de instituciones los procedimientos son poco claros y escasas aclaraciones a las consultas, dando pie a sacar la vuelta al estado con serios actos de corrupción que al final son sancionados por el órgano de control interno. Respecto a las contrataciones con el estado, los autores coinciden en que están enmarcadas en la ley respectiva, con el fin de 
hacer los procesos de compras más eficientes y evitar la corrupción. Sin embargo, los procesos vinculantes no están siendo muy claros, ya que da pie a que sean analizados de manera diferente por las entidades; por ello, las mismas deben ser estandarizados e institucionalizados para ser aplicados por igual para todos, con procesos claros y sencillos. A su vez, los procesos de contrataciones están estipulados en la ley de contrataciones de manera general, con derechos y obligaciones de las partes, con el fin de ayudar a la eficiencia de procesos de compras, las mismas que no funciona a cabalidad en las instituciones públicas por el deficiente control interno en las mismas.

Por ello, se puede decir que el control interno es débil en las instituciones públicas, los procedimientos en cuanto a las contrataciones en compras y licitaciones no se desarrollan a cabalidad, a pesar de tener un aspecto vinculante, debido a que lo procedimientos son poco claros y confusos, llevando a que el órgano de control tenga que llegar a sancionar a las instituciones. Es decir, falta estandarizar los procesos y hacerlos más sencillos, con el objetivo de que las instituciones cumplan los objetivos usando los recursos de manera eficiente.

\section{REFERENCIAS}

Becerra, M. (2016). El tribunal de contrataciones del estado en el periodo 2012-2014. Revista Derecho \& Sociedad, (44), 353-363, Perú. Recuperado de: http://revistas.pucp.edu.pe/index.php/derechoysociedad/article/view/14417/15031

Castillo, M., y Sabroso, M. (2019). Los árbitros y el deber de revelación en la ley de contrataciones del estado y su reglamento. Revista Derecho \& Sociedad, (44), 245255, Perú. Recuperado de: http://revistas.pucp.edu.pe/index.php/derechoysociedad/article/view/14408/15022

Chacón, R. (2018). Implicancia de la auditoría integral y su sostenibilidad procedimental teórica practica en las empresas de servicios de saneamiento para obtener eficacia eficiencia efectividad en la dirección estratégica corporativa. (Tesis de posgrado, Universidad Nacional Federico Villareal), Lima, Perú. Recuperado de: http://repositorio.unfv.edu.pe/bitstream/handle/UNFV/2421/CHAC\%C3\%93N\%20 VEGA\%20\%20ROGERIO\%20MANUEL.pdf?sequence=1\&isAllowed=y

Cuevas, L. (2018). Control interno del sector público. Comisión de Contabilidad y Auditoría Gubernamental del Colegio de Contadores Públicos de México. 
Recuperado

de:

https://www.ccpm.org.mx/avisos/2018-

2020/control.interno.cofi.pdf

Effio, A. (2015). La falsa reforma de la normativa de contrataciones del estado: Instituciones, incertidumbre y vaguedades. Revista Intuiciones, Incertidumbre y Vaguedades. Derecho \& Sociedad, (44), 109-119. Recuperado de: http://revistas.pucp.edu.pe/index.php/derechoysociedad/article/view/14393

Franco, B. (2015). El carácter vinculante de las opiniones del organismo supervisor de las contrataciones del estado. Revista Derecho \& Sociedad, (44), 391-402, Perú. Recuperado de: http://revistas.pucp.edu.pe/index.php/derecho\%20ysociedad/article/view/14420

Gamboa, J., Puente, S., e Ysidora, P. (2016). Importancia del control interno en el sector público. Revista publicando, 3(8). 487-502. Universidad de Guayaquil. Recuperado de: https://dialnet.unirioja.es/servlet/articulo?codigo=5833405

Huamán, C., \& Jiménez, B. (2014). Diseño del sistema de control interno del área de tesorería Para promover eficiencia operativa, en la Municipalidad Distrital de Morales año 2014. (Tesis de posgrado, Universidad Nacional de San Martín). Tarapoto, Perú. Recuperado de: http://repositorio.unsm.edu.pe/handle/11458/1238

Leiva, D. y Soto, H. (2015). Propuestas de políticas para los gobiernos regionales 2015 2018. Control Interno como herramienta para una gestión pública eficiente y prevención de irregularidades. Consorcio De Investigación Económica Y Social (CIES). Lima. Recuperado de: http://www2.congreso.gob.pe/sicr/cendocbib/con4_uibd.nsf/97D59BF5DEC57F79 05257F870071C2AA/\$FILE/021-daniel_leiva-hugo_soto.pdf

Mendoza, W., García, T., Delgado, M., y Barreiro, I. (2018). El control interno y su influencia en la gestión administrativa del sector público. Revista Dom. Cien, Vol. 4, Núm. 4, pp 206-240. Recuperado de: https://dialnet.unirioja.es/servlet/articulo?codigo $=6656251$

Organismo Supervisor de Contrataciones con el estado - OSCE (2019). Plan anual de contrataciones del año 2019. Lima, Perú. Recuperado de: https://cdn.www.gob.pe/uploads/document/file/562579/Plan_Anual_de_Contrataci ones_del_a\%C3\%B1o_2019.pdf 
Pedreschi, W. (2015). Aproximaciones al régimen de contrataciones directa en la nueva ley de contrataciones del estado. Revista Derecho \& Sociedad, (44), 163-174. Recuperado de: revistas.pucp.edu.pe > index.php > article > download

Perdomo, A. (2004). Fundamentos del control interno. Ed. $\left(1^{\circ}\right)$, International Thomson Editores, México, $318 \mathrm{pp}$. Recuperado de: https://books.google.com.pe/books/about/Fundamentos_de_control_interno.html?id $=$ VobCCBsMJtoC

Rivas, F., Asprino, M., Sarache, J., y otros (2019). Sistema inteligente para la generación automática de contratos en el marco de la ley de contrataciones públicas. Revista Politécnica, Vol. 44, No. 1, Ecuador. Recuperado de: https://doi.org/10.33333/rp.vol44n1.05

Romero, J. (31 de 08 de 2012). Control Interno y sus 5 componentes según COSO. Geopolis. Recuperado de: https://www.gestiopolis.com/control-interno-segun-coso/ Transparency internacional (2018). Contrataciones Públicas en América Latina: Instituciones, prácticas y riesgos de corrupción. Estudio del riesgo en los sistemas de las contrataciones públicas en 9 países. [Informe mundial de contrataciones]. Recuperado de: http://www.osce.gob.pe/boletininstitucional/informe\%20regional_contrataciones_s $\mathrm{p}[1] . p d f$

Zegarra, J. (2015). Modificaciones al contrato en la normativa de contrataciones del estado (Ley 30225). Revista Derecho \& Sociedad, N 44 / pp. 191-202, Perú. Recuperado de:

http://revistas.pucp.edu.pe/index.php/derechoysociedad/article/view/14401/15016 